\title{
EFFECT OF PHOTON ENERGY ON THE RESPONSE OF MOS DEVICES
}

\author{
C. M. Dozier and D. B. Brown \\ Naval Research Laboratory \\ Washington, D. C. 20375
}

\begin{abstract}
Radiation-produced flatband voltage shifts in MOS capacitors have been measured as a function of incident photon energy and applied electric fields with UV, $x$ - and gamma-ray sources spanning the energy range $70 \mathrm{eV}$ to $1.25 \mathrm{MeV}$. Special interest was directed to the energies below $20 \mathrm{keV}$ where the greatest effects on the flatband voltages were expected. At $70 \mathrm{eV}$ the shifts are almost as great as those observed at $1.25 \mathrm{MeV}$ $\left({ }^{60} \mathrm{Co}\right)$. For $1.49 \mathrm{keV}$ incident photons the voltage shifts are less than $1 / 3$ those observed for ${ }^{60} \mathrm{Co}$ photons.
\end{abstract}

\section{INTRODUCTION}

The importance of incident photon or particle energy and electronhole recombination dynamics and on radiation effects in MOS devices has been noted by many investigators. ${ }^{1-5}$ While most investigators have not been concerned with effects caused by source energy, the data referenced indicate that recombination for low energy $(\leqslant 10 \mathrm{keV})$ ionizing radiation is greater than that for much higher energies $(\geqslant 500 \mathrm{keV})$. The relative magnitude of these effects in MOS devices has been determined only for the energies between $5-10 \mathrm{keV}$ and energies in excess of 1 $\mathrm{MeV}^{3,5}$ The current interest in uses of low energy $\mathrm{x}$-ray sources in electronics, e.g. lithography and "on-chip" testing, increase the need for a more complete knowledge of the interrelation of recombination, incident photon or particle energy, and applied electric field.

The understanding of the details of electron-hole recombination dynamics is still somewhat rudimentary. For example, the magnitude of the effects as a function of energy, and the role impurities and structural defects are known for only a few materials, such as the noble gases. ${ }^{6}$ Not only is the experimental knowledge incomplete, but the models which have been applied to the problem are not entirely satisfactory. In describing recombination in electronic materials, for example, two models have commonly been used. One model ${ }^{1}$ predicts that recombination should be temperature dependent although this behavior has not been observed experimentally. ${ }^{7}$ The second model $^{8}$ has been used successfully to describe the response of MOS capacitors irradiated by low energy electrons, but fails to be physically reasonable at high energies. The solutions to these and other problems are important not only to electronics but also to a number of other fields such as radiation chemistry and biology.

To begin to understand radiation deposition requires the recognition that the deposition is a cascade process. The incident photon or ionizing particle interacts with materials to produce secondary electrons through excitation or scattering processes. These secondary electrons, in turn, may repeat the process until all of the energy is dissipated. Although these steps cannot be followed easily in experiments, a few theoretical models ${ }^{9-11}$ have been developed in an effort to describe the processes more completely. One model, developed by Magee and coworkers ${ }^{10,11}$ for radiation effects in chemical systems, suggests that effects dependent on the energy of the incident photons should be expected and predicts that experimentally observable differences should occur at low energies. However, this model has never been fully tested. The characteristics of the model of Magee et al. suggests that the basic ideas also should be applicable to recombination in $\mathrm{SiO}_{2}$. The properties of MOS capacitors are such that they can be used to measure the magnitude of the energy effects and to verify more convincingly the validity of the model.

This paper is an extension of earlier work on energy effects on recombination in MOS structures. ${ }^{5}$ In it we present results of experimental measurements which provide additional evidence of the magnitude of the recombination effects in $\mathrm{SiO}_{2}$ in the energy range of $70 \mathrm{eV}$ to $1.25 \mathrm{MeV}$. In addition, an effort has been made in the current experiments to measure not only the magnitude of the energy effects expected in electronic systems but also to determine what processes are involved in recombination. Therefore, the selection of energies was made in light of the predictions of the model of Magee et al. Only a brief description of the details of the model are given in this paper. If the reader has further interest in the physical mechanisms, he is directed to a companion paper ${ }^{12}$ where the authors apply a model based on the work of Magee and coworkers, ${ }^{10,11}$ derived for chemical systems, to $\mathrm{SiO}_{2}$.

\section{THE EXPERIMENTAL PROCEDURES}

In these experimental measurements the fraction of recombination in $\mathrm{SiO}_{2}$ was determined for various incident photon energies. The fraction at each energy was inferred from the flatband voltage measurements of MOS capacitors irradiated at several positive biases. With a field across the capacitor during irradiation, holes escaping recombination are swept to the $\mathrm{Si}: \mathrm{SiO}_{2}$ interface where some are trapped. At low applied fields during irradiation electron-hole recombination dominates, and only a small fraction of the holes produced by the ionizing radiation are swept to the interface. At very high fields, on the other hand, the imposed field dominates and essentially all the holes produced are separated from the electrons and are swept to the interface. The shift in the flatband voltages caused by the holes trapped provide a measure of the total number of holes which reach the interface region. It will be shown later in the paper how the flatband voltage shifts at several fields and for several incident energies can elucidate recombination dynamics.

MOS capacitors fabricated on a single $3-5 \mathrm{ohm}$-cm n-type (100) Czochalski-grown $\mathrm{Si}$ wafer were used for the measurements. Wet oxides were grown $500 \mathrm{~nm}$ thick. The relatively thick oxides were chosen to maximize the rate of shift in the flatband voltage for the measurements at $1.49 \mathrm{keV}$ where a relatively weak $\mathrm{x}$-ray source was used. $\mathrm{Al}$ dots, 0.75 $\mathrm{mm}$ diameter and $200 \mathrm{~nm}$ thick, completed the MOS structures. Thirteen capacitors cut from the wafer were mounted on 14-pin DIP base; one pin served as a common connector to the Si substrate. Biases during irradiation ranged from approximately $-0.3 \mathrm{~V}$ to $+250 \mathrm{~V}$ (fields of approximately $1 \times 10^{3}$ to $5 \times 10^{6} \mathrm{~V} / \mathrm{cm}$ ).

\section{THE SOURCES}

The sources used to irradiate the capacitors are described briefly below. Figure 1 shows schematically the experimental setups, and Table 1 lists parameters for these sources.

SURF: Figure 1a. Monochromatic $70 \mathrm{eV}$ photons of synchrotron radiation were obtained from the $243 \mathrm{MeV}$ electron storage ring located at the National Bureau of Standards (NBS). The electrons, accelerated centripetally within an electron storage ring, emit continuous radiation tangentially to the ring from the IR into the UV range with a maximum photon energy of approximately $200 \mathrm{eV}$. Samples were mounted in an ultrahigh vacuum chamber almost 10 meters from the ring. The photon energy of interest for the experiment was selected with a toroidal grating monochromator. Between the monochromator and the sample location a $50 \mathrm{~cm}$ long capillary tube, $0.6 \mathrm{~mm}$ in diameter, collimated the beam further. As a result, only one sample could be irradiated at a time. Scattering and reflection within the collimator tube caused the beam to expand to a little over $0.75 \mathrm{~mm}$ at the sample position. This made tolerances very small. However, alignment problems were alleviated somewhat by the ability to use visible light for alignment. Initial alignment was made on several capacitors which were subsequently unused for radiation effects measurements. And following each exposure series, the alignment was checked again visually, and adjustments made to improve the alignment on the next capacitor. Only those results from samples that were well centered on the dots are included in the results.

VACUUM SPECTROMETER: Figure $1 \mathrm{~b}$. The $1.49 \mathrm{keV}$ radiation was obtained by fluorescing the $\mathrm{K} \alpha$ line of a $15 \mu \mathrm{m} \mathrm{Al}$ foil with a $\mathrm{Cr} \mathrm{x}-$ ray tube operated at $45 \mathrm{kV}$ and $45 \mathrm{ma}$ in a Phillips Model 1410 vacuum spectrometer. The use of a thin foil fluorescer reduced the scattering of the $\mathrm{Cr}$ tube spectrum onto the MOS capacitors. Further reduction in the scatter contribution was achieved by eliminating the view of the vacuum housing walls with a blade collimator. The $\mathrm{Al} \mathrm{K} \alpha$ photons produced over 80 percent of the dose in the $\mathrm{SiO}_{2}$. The MOS capacitors were mounted at the position normally occupied by a crystal and irradiated while viewing 


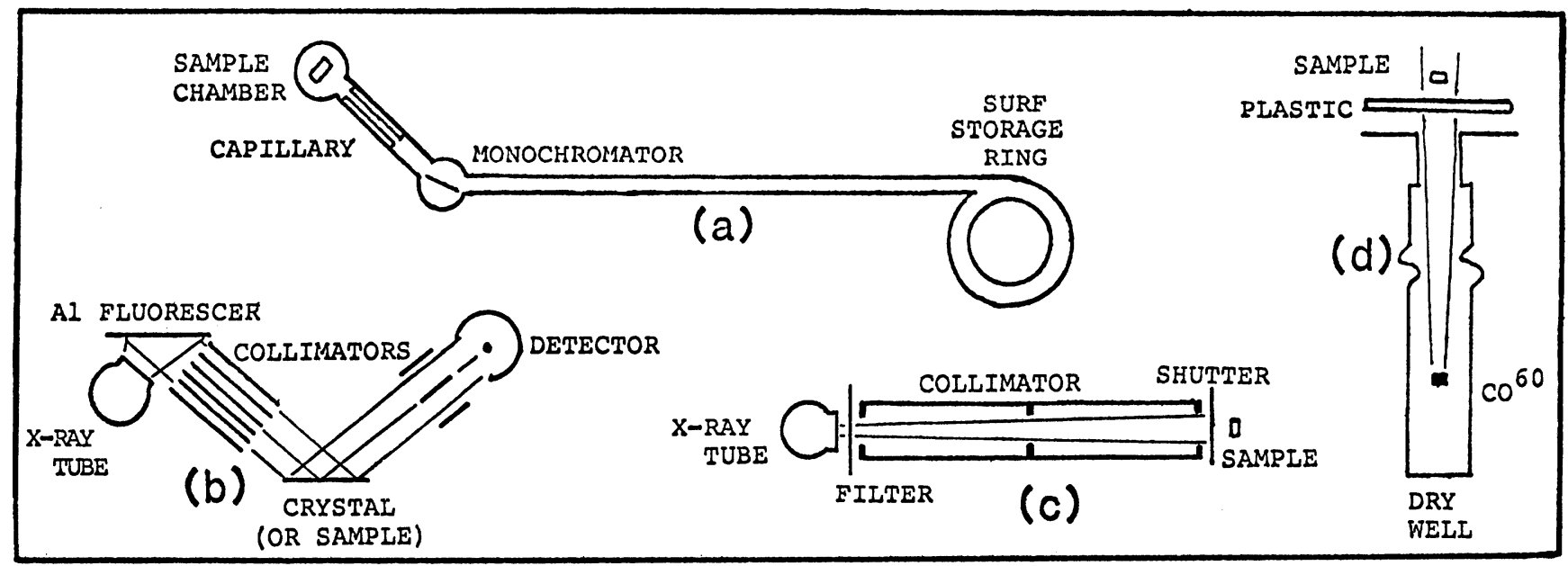

Fig. 1 - Schematic drawings of the four types of sources used for irradiation. Part (a) is the synchrotron radiation source, SURF, at the National Bureau of Standards. Part (b) shows the $\mathrm{x}$-ray tube excited fluorescer which was used to generate the Al spectrum. Part (c) shows the setup which was used for $\mathrm{Cu}$ target and Mo target $\mathrm{x}$-ray tubes. Part (d) shows the geometrical arrangement of the ${ }^{60} \mathrm{Co}$ source used.

Table I - Radiation Sources

\begin{tabular}{|c|c|c|l|}
\hline Source & Type & $\begin{array}{c}\text { Photon } \\
\text { Energy }\end{array}$ & \multicolumn{1}{|c|}{ Comments } \\
\hline Sl & Fluorescer & $1.49 \mathrm{keV}$ & $\begin{array}{l}\text { Synchrotron } \\
\text { Excited by } \mathrm{Cr} \\
\text { x-ray tube operated at } \\
45 \mathrm{kV}, 45 \mathrm{ma}\end{array}$ \\
$\mathrm{Cu}$ & $\mathrm{X}$-ray tube & $8.04 \mathrm{keV}$ & $\begin{array}{l}\text { Emission of } 243 \mathrm{MeV} \\
\text { Operated at } 45 \mathrm{kV}, \\
2.5 \text { ma }\end{array}$ \\
$\mathrm{Mo}$ & $\mathrm{X}$-ray tube & $17.4 \mathrm{keV}$ & $\begin{array}{l}\text { Operated at } 45 \mathrm{kV}, 4 \mathrm{ma} \\
\text { Dose from } 15-20 \mathrm{keV} \text { band }\end{array}$ \\
$\mathrm{Co}^{60 *}$ & Isotope & $1.25 \mathrm{MeV}$ & $\begin{array}{l}\text { Located in dry } \\
\text { well having some } \\
\text { collimation }\end{array}$ \\
\hline
\end{tabular}

*Data from Reference 5

the fluorescer directly. Three micron aluminized mylar covered both the samples and dosimeters to eliminate the effects of room light.

X-RAY TUBES: Figure 1c. The $\mathrm{Cu}(8.04 \mathrm{keV})$ and Mo (17.4 $\mathrm{keV})$ exposures were obtained with Machlett OEG-50 spectrographic $\mathrm{x}$ ray tubes operated at $45 \mathrm{kV}$ and 2.5 and $4.7 \mathrm{ma}$, respectively. Each tube window was covered with a K-edge filter of the appropriate thickness to eliminate most of the $K \boldsymbol{\beta}$ line and a substantial fraction of the continuum radiation. For the $\mathrm{Cu}$ tube the $\mathrm{K}$-edge filter eliminated most of the low and high energy portions of the continuum and approximately 80 percent of the dose in the $\mathrm{SiO}_{2}$ was produced by the $\mathrm{Cu} \mathrm{K} \alpha$ line. By comparison, the filtering was not as good for the Mo tube where only 47 percent of the dose was from the $K \alpha$ line. However, approximately 90 percent of the dose was produced by the $\mathrm{K} \alpha$ line and continuum radiation between 15 and $20 \mathrm{keV}$. Increasing the filtration reduced the intensity of the beam from the Mo tube without improving the relative line to continuum ratio in the dose. The MOS capacitors were located approximately 1 meter from the tube targets at the end of a collimator-shutter assembly and were shielded from room light by 3 micron thick aluminized Mylar.

${ }^{60} \mathrm{Co}$ : Figure 1d. Results shown as those for ${ }^{60} \mathrm{Co}$ are taken from our work reported a year ago. ${ }^{5}$ In that study, we obtained results for three different samples all of which showed that approximately 1.8 times as much recombination occurred for $\mathrm{Cu}$ irradiations as did for ${ }^{60} \mathrm{Co}$ irradiations. The measurements were not repeated since consistent results had been obtained already for that source. Therefore, we have scaled the
$\mathrm{Cu}$ data from the present work by a factor of 1.8 in order to obtain a value for ${ }^{60} \mathrm{Co}$. For completeness in this paper it should be noted that in the earlier measurements the ${ }^{60} \mathrm{Co}$ data was gathered at one of NRL's dry ${ }^{60} \mathrm{Co}$ wells. Some collimation was provided by the well design, and a 1.27 $\mathrm{cm}$ thick plastic window covered the well to stop electrons. During exposures the capacitors were surrounded by $\mathrm{Al}$ coverings sufficiently thick to insure that "equilibrium" conditions for photoelectrons generated in the samples were established.

\section{DOSIMETRY}

Because of the number of different sources, energies and experimental geometries used, quantitative comparison between the results required that care be taken in the dosimetry measurements. Photodiodes, $\mathrm{CaF}_{2}$ TLD chips, spectral measurements and calculations were used to insure the accuracy of the dosimetry. A detailed description of the procedures for using $\mathrm{CaF}_{2}$ dosimeters have been described elsewhere $e^{4,5}$. Below we summarize the steps taken. Table II contains results of the measurements and calculation of doses in experiments where the quality of the TLD measurements were checked.

The incident intensity at $70 \mathrm{eV}$ was determined using an NBScalibrated standard photodiode. Dosimetry at $70 \mathrm{eV}$ with TLD's is not an effective technique due to high surface dose and dose-rate effects in the TLD materials for UV radiations ${ }^{13}$ and due to the small spot size and the limited access to components in the ultrahigh-vacuum chamber. Quantum efficiencies for photo-emission from the photocathode have been measured to be 6.5 percent at $70 \mathrm{eV}$, and Figure 2 shows the calculated deposition profile for $70 \mathrm{eV}$ photons through the capacitor. Approximately 80 percent of the incident intensity is transmitted through the $200 \mathrm{~nm} \mathrm{Al}$ dots. However, in $\mathrm{SiO}_{2}$ the beam is rapidly attenuated, and essentially all of the deposition occurs within the first $100 \mathrm{~nm}$ of the oxide.

At the normal electron currents in the storage ring, the intensities were too high for experiments that would be comparable to those at higher photon energies. By running with beam intensities two to three orders of magnitude lower than typically available, dose rates in a suitable range could be obtained. The dose rate at the front surface of the $\mathrm{SiO}_{2}$ ranged between approximately $40-185 \mathrm{rads}\left(\mathrm{SiO}_{2}\right) / \mathrm{sec}$. A few runs at lower dose rates indicated that dose-rate effects probably were not a problem.

Dosimetry for the $\mathrm{Al}, \mathrm{Cu}$, Mo and ${ }^{60} \mathrm{Co}$ used included $\mathrm{CaF}_{2}$ TLD chips wrapped in an equilibrium thickness of $\mathrm{Al}$ and placed just ahead of, but not shielding, the samples. For the $\mathrm{Al}, \mathrm{Cu}$, and Mo energy sources the spectra of the tubes were measured experimentally; for the $\mathrm{Cu}$ and Mo x-ray tubes they were also calculated. To check the TLD results, the dose in the TLD's was calculated using the spectral distribution and a deposition code ${ }^{14}$ and found to agree within 10 percent. Corrections for 
Table II - Dosimetry

\begin{tabular}{|c|c|c|c|c|}
\hline Energy & Dosimeter & $\begin{array}{c}\text { Measured } \\
\text { Doserate }\end{array}$ & $\begin{array}{c}\text { Calculated } \\
\text { Doserate }\end{array}$ & $\begin{array}{c}\text { Dose Rate } \\
\text { in Oxide }\end{array}$ \\
\hline \hline $70 \mathrm{eV}$ & $\begin{array}{l}\text { NBS-calibrated } \\
\mathrm{Al}_{2} \mathrm{O}_{3} \text { Photodiode }\end{array}$ & $4.0 \times 10^{7} \mathrm{ph} / \mathrm{sec}^{*}$ & - & $\begin{array}{c}4.0 \times 10^{1} \\
\left(1.8 \times 10^{2}\right)^{* *}\end{array}$ \\
$1.49 \mathrm{keV}$ & $\mathrm{CaF}_{2}$ TLD Chips & $7.0 \times 10^{-4}$ & $6.9 \times 10^{-2}$ & \\
$8.04 \mathrm{keV}$ & $\mathrm{CaF}_{2}$ TLD Chips & $8.8 \times 10^{-2}$ & $8.3 \times 10^{-2}$ & $2.6 \times 10^{-1}$ \\
$17.4 \mathrm{keV}$ & $\mathrm{CaF}_{2}$ TLD Chips & $1.8 \times 10^{-1}$ & $1.7 \times 10^{-1}$ & $2.1 \times 10^{-1}$ \\
$1.25 \mathrm{MeV}$ & $\mathrm{CaF}_{2}$ TLD Chips & $6.7 \times 10^{-2}$ & - & $7.0 \times 19^{-2}$ \\
\hline
\end{tabular}

${ }^{*}$ Dose was not measured. The value is the photon flux for a 5 ma current in the SURF electron storage ring.

${ }^{* *}$ Value in parentheses is the maximum dose rate at the front surface of the MOS capacitor oxide.

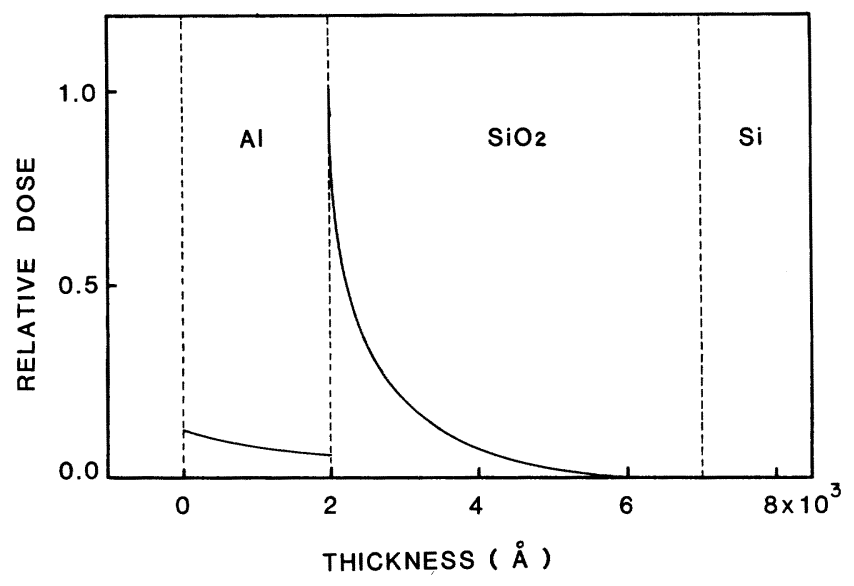

Fig. 2 - Energy deposition profile produced in our capacitors by the 70 $\mathrm{eV}$ radiation from SURF.

dose enhancement due to interface effects in the MOS capacitors were made based on reference 15 . This resulted in an increase in dose of 30 percent for $\mathrm{Cu}$ and 15 percent for Mo. At the operating voltages and currents used, the dose rates in the MOS oxides were 0.26 and 0.21 $\operatorname{rads}\left(\mathrm{SiO}_{2}\right) / \mathrm{sec}$ for the $\mathrm{Cu}$ and $\mathrm{Mo}$ tubes respectively.

In the $\mathrm{Al}$ experiment the cross checks on the intensity required additional steps to those made at the higher energies. The intensity of the direct beam could not be measured with the detector in the spectrometer. Therefore measurement of the intensity and the spectral distribution was made after diffraction of the $x$-rays by a crystal. The spectra consisted of primarily the Al K $\alpha$ line with a small contribution from scattered lines and continuum. Collimators and the large nonuniform fluoresced region on the Al complicated the geometrical corrections. However, a calculation, after correcting for the diffraction efficiency of the crystal ${ }^{16}$, agreed to within 35 percent of the values measured with the dosimeters. This result is somewhat worse than we hoped for, but due to the complex geometry within the spectrometer the agreement is probably satisfactory. The corresponding dose rate in $\mathrm{SiO}_{2}$ was $0.07 \mathrm{rads} / \mathrm{sec}$.

\section{EXPERIMENTAL RESULTS}

Typical C-V curves for $70 \mathrm{eV}$ irradiations at SURF, which received the highest doses of any samples, are shown in Figure 3. Maximum flatband voltage shifts just over $2 \mathrm{~V}$ were observed at the highest applied fields. As can be seen in the figure the shapes of the curves are essentially parallel, indicating that little of the observed shifts is due to surface states. Similarly shaped curves were obtained for samples irradiated at higher energies.

Flatband voltage shifts obtained from data at other fields were plotted as a function of dose as shown in Figure 4. Only the data for fields greater than $1 \times 10^{4} \mathrm{~V} / \mathrm{cm}$ are included since diffusion processes may

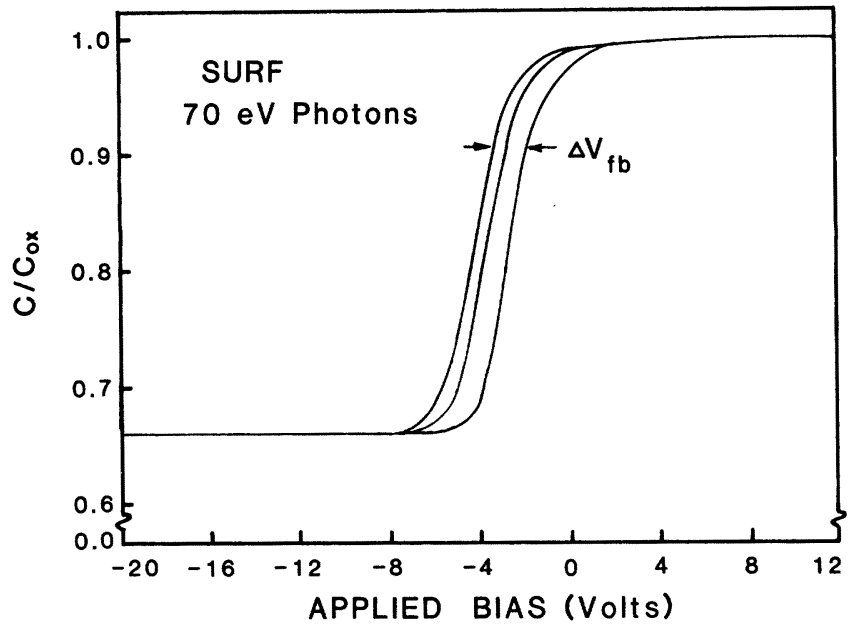

Fig. 3 - examples of the C-V curves generated in the SURF experiment.

begin to affect the recombination behavior at lower fields. These data have been normalized using an equation of the following form:

$$
\Delta V_{f b}(\text { Normalized })=\frac{\Delta V_{f b}}{\left[\int \text { (Dose) } d t\right] \text { [thickness] }}
$$

On the right hand side of the equation, the numerator is the observed flatband voltage shift. In the denominator the first term is a proportional to the number of electron hole pairs produced within the oxide volume. The second term in the denominator allows for the fact that the voltage measured on a parallel plate capacitor is inversely proportional to the separation of the two plates. In Figure 4 it can be seen that, at high fields, where the applied field dominates the recombination processes and essentially all charges escape recombination, the curves approach asymptotes which are indicated as dashed lines. The dashed lines in Figure 4 represent the flatband voltage shift which would occur if there were no electron-hole loss due to recombination. It will be observed that this depends on field approximately as $E^{-1 / 2}$. We have previously discussed such behavior in Reference 5 where it is argued that the asymptote represents a field dependence of the trapping at the $\mathrm{Si}: \mathrm{SiO}_{2}$ interface. The fraction of electron-hole pairs which recombine at each value of field is taken as the ratio of the actual measured trapped charge (solid line) to the trapped charge in the absence of recombination (dashed line).

The positioning of the dashed lines on the plots in Figure 4 was determined by a technique suggested by the work of Ausman and McLean. ${ }^{8}$ In their paper the reciprocal of the field versus the reciprocal of the collected charge were plotted to determine an average energy of formation of an electron hole pair in $\mathrm{SiO}_{2}$. We have plotted the reciprocal of the field versus the reciprocal of the fraction of electron-hole pairs unrecombined, adjusting the dashed lines of Figure 4 until at infinite field the fraction of electron-hole pairs which are unrecombined is unity. Figure 5 shows the inverse fraction of electrons which escape recombina- 


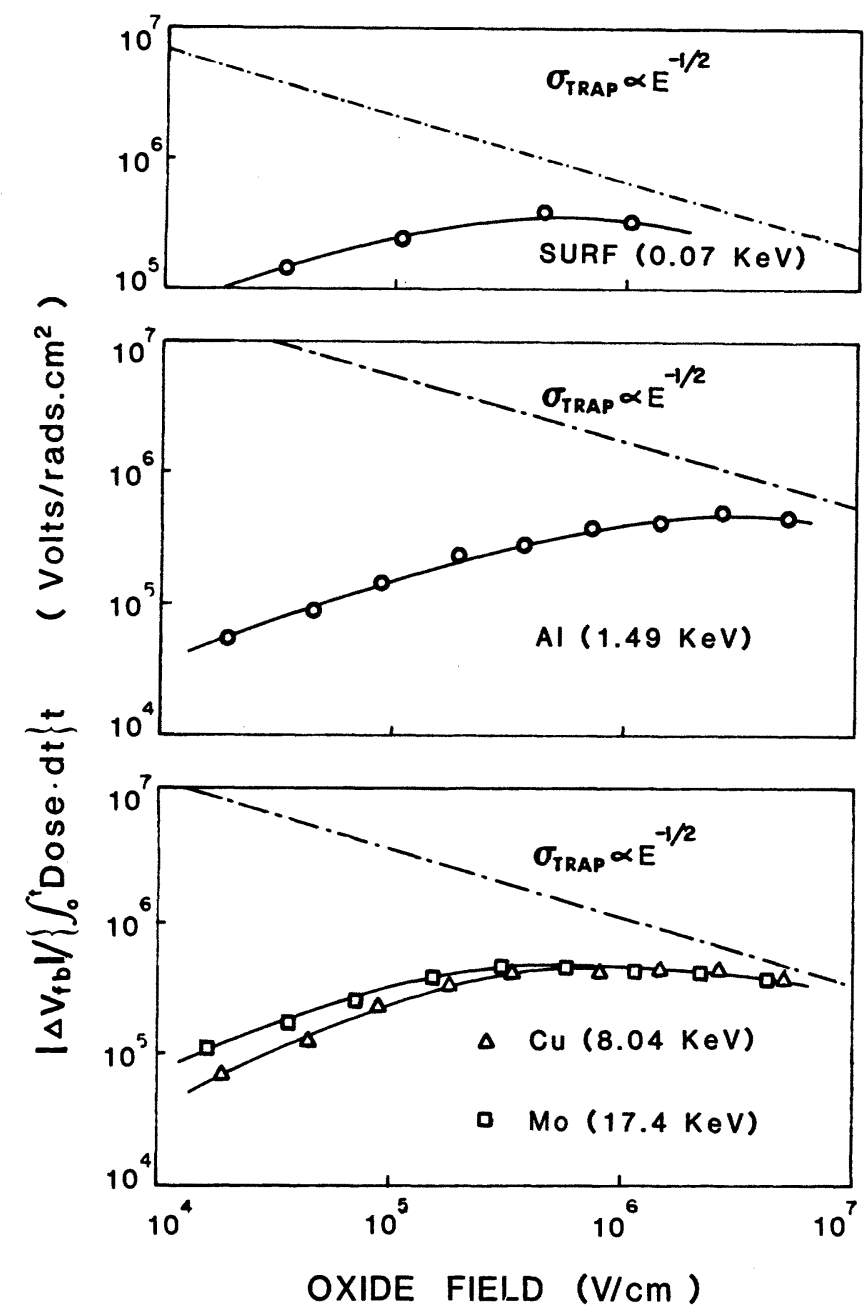

Fig. 4 - Normalized flat band shift data for $0.07,1.49,8.04$, and 17.4 kev (nominal) irradiation. The dashed lines represent the flat band shift which would have been obtained if there had been zero recombination. These dashed lines show a field dependence of approximately $\mathrm{E}^{-1 / 2}$. This is believed to be caused by a field dependence of trapping at the $\mathrm{Si}: \mathrm{SiO}_{2}$ interface, as discussed in the text.

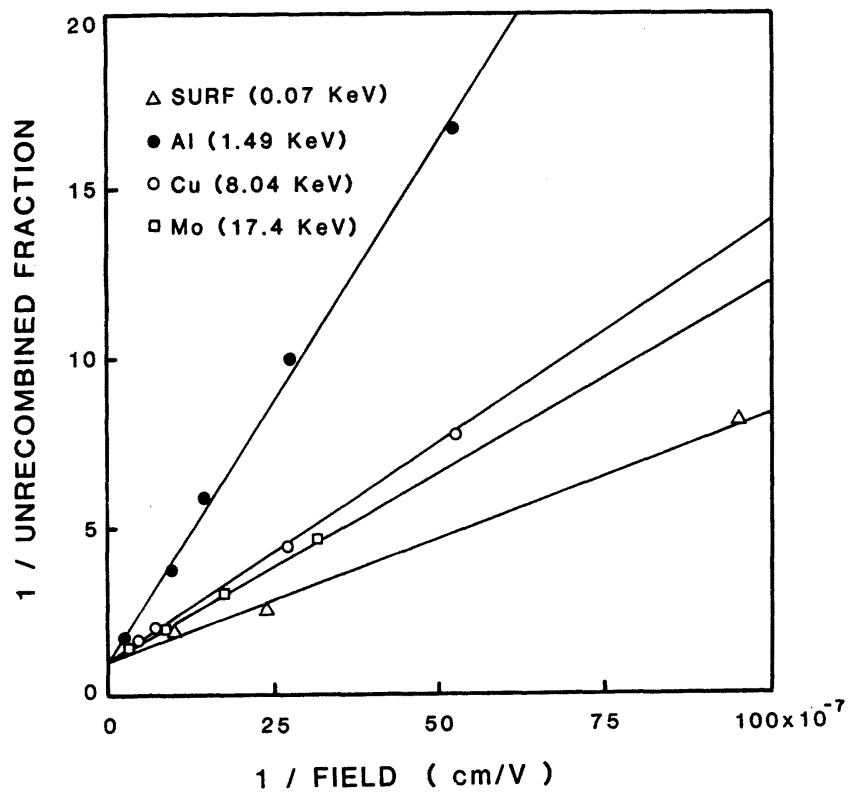

Fig. 5 - A plot of the reciprocal of the unrecombined fraction of electron-hole pairs versus the reciprocal of the field in the oxide. Note that the data can be fit by a straight line which goes to one at infinite field.

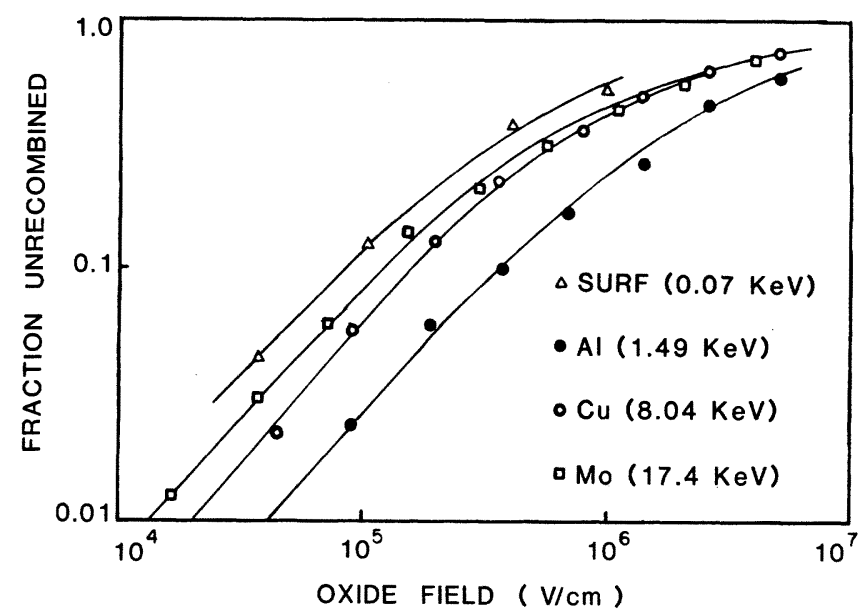

Fig. 6 - A plot of the fraction of unrecombined electron-hole pairs versus field in the oxide for $0.07,1.49,8.04$, and $17.4 \mathrm{kev}$ (nominal) irradiation

tion as a function of the inverse applied field. It can be seen that all the curves have a linear form that Ausman and McLean observed. Figure 6 is then a plot of this data in a form where the fraction of the unrecombined charge can be obtained directly as a function of the applied field. The fractions are similar to results obtained by many other experimenters. ${ }^{1-5.7}$

\section{DISCUSSION}

Earlier in the paper we stressed the dosimetry necessary in order to intercompare results obtained from several sources of rather different spectral characteristics. The agreement between doses measured with TLD's and those calculated in $\mathrm{CaF}_{2}$ using the spectral distributions for the $\mathrm{x}$-ray tubes was within \pm 10 percent for the $\mathrm{Cu}$ and Mo experiments. (See Table II) These results are within the experimental accuracies of TLD's when used in a "batch" mode. For the SURF and Al data, on the other hand, the overall estimated errors are \pm 50 percent. Much of the contributions to the errors in these doses is due to geometrical uncertainties. Additionally, in the case of the SURF data, the value for the energy of formation of electron-hole pairs in $\mathrm{SiO}_{2}$ is uncertain. Work on gases such as argon ${ }^{6}$ indicate that at $70 \mathrm{eV}$ the average energy of formation has increased by approximately 20 percent. We have included a 20 percent correction to the data of Figure 4; however, it is not clear that this correction to the value for the energy of formation of electron-hole pairs in $\mathrm{SiO}_{2}$ is correct. Fortunately, the adjustment to make the recombination zero in the limit of very high fields, as shown in Figure 5, reduces the relative errors in the data. Therefore, we estimate the relative errors in the unrecombined fraction in Figure 6 are within \pm 20 percent.

Figure 6 shows that the recombination of electrons and holes for the several incident photon energies is not the same. In Figure 7 the fraction of unrecombined charges are plotted for a field of $3 \times 10^{5} \mathrm{~V} / \mathrm{cm}$. Included in Figure 7 is a curve based on the theoretical calculations of Brown and Dozier ${ }^{15}$ for electron-hole recombination behavior in $\mathrm{SiO}_{2}$. In those calculations, we predict the recombination based on electron energies. In this paper we adjust electron energies to photon energies assuming that the electron energy in the calculations is that of the primary photoelectron or Compton electron. For $70 \mathrm{eV}$ photons the correction is approximately $10 \mathrm{eV}$; for $1.25 \mathrm{MeV}$ photons the correction is approximately $500 \mathrm{keV}$.

The dip in the data and calculations is attributed to recombination of several different kinds of deposition mechanisms. For very low energy photons $(\geqslant 150 \mathrm{eV})$, the interactions produce low energy delta rays with insufficient energies to escape the Coulombic attraction of the initial parent positive charge center, and the electron-hole production occurs in a very small region surrounding the parent charge center. At yet higher energies ( $\geqslant 150 \mathrm{eV}$ to about $5 \mathrm{keV}$ ), the delta rays can escape the ionic center. In addition, about half of the energy loss occurs by resonant interactions which produce plasmons and excitons. The energy deposited in such interactions is less than $100 \mathrm{eV}$. For energies of 150 $\mathrm{eV}$ to $5 \mathrm{keV}$ the linear energy transfer (LET) is sufficiently high that 


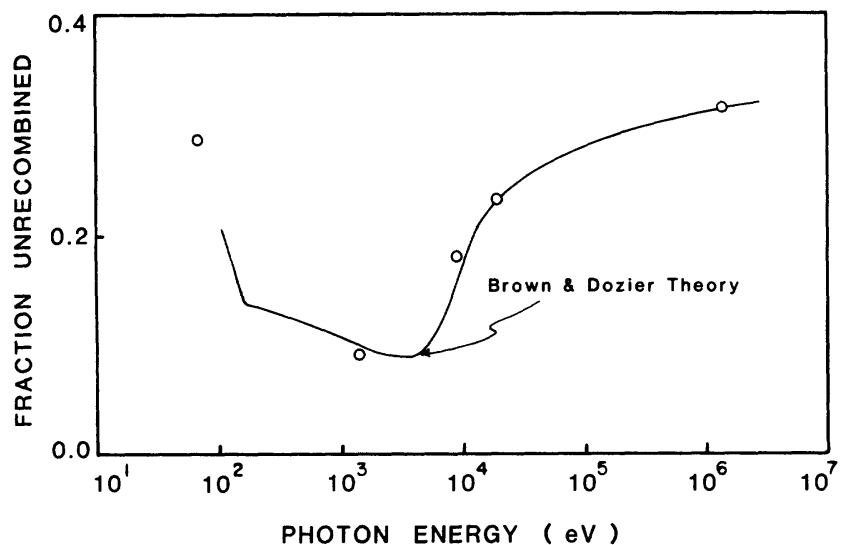

Fig. 7 - A plot of the fraction of unrecombined electron-hole pairs versus incident photon energy for a field of $3 \times 10^{5} \mathrm{~V} / \mathrm{cm}$. The solid line represents the calculation of Brown and Dozier. ${ }^{12}$

these low energy losses are close together, resulting in electron-hole pairs which are close enough together that the electrons may combine with holes other than their parent hole (columnar recombination). At energies above approximately $5 \mathrm{keV}$ the low energy losses are no longer close together, resulting in relatively isolated electron-hole pairs. Thus both at very low energies $(\leqslant 100 \mathrm{eV})$ and at high energies $(\geqslant 100 \mathrm{keV})$ electron-hole pairs are spatially relatively isolated. It follows that low energies should produce nearly the same results as those observed with ${ }^{60} \mathrm{Co}$ radiation.

The results of this study have implications for applications where UV or low energy $x$-ray sources may be used. For $x$-ray lithography, photon sources in the energy range of $100 \mathrm{eV}$ to approximately $3 \mathrm{keV}$ are being considered. It appears that in this energy region that there will be less damage to under-lying oxide layers than have been predicted based on ${ }^{60} \mathrm{Co}$ irradiation data. Additionally, where quantitative comparisons are to be made between data obtained using low and high energy sources, corrections for the response with energy of MOS devices may be required. All of the results here are at relatively low doses. Further work is needed to determine whether or not photon energy influences the production of surface states and bulk trapping.

\section{CONCLUSIONS}

Recombination dynamics of electrons and holes in MOS structures vary with incident photon energy. The differences in the recombination can be seen as a minimum in the shifts of flatband voltage which occurs in the $100 \mathrm{eV}$ to $3 \mathrm{keV}$ region. The recombination at $1.49 \mathrm{keV}$ (at or near the minimum in the data) is approximately one third or less or the recombination observed for ${ }^{60} \mathrm{Co}$ at the same field. The experimental data fit the calculations of Brown and Dozier well. This implies that the model is a reasonably good description of general recombination behavior in $\mathrm{SiO}_{2}$. It may be necessary to include energy dependent effects when quantitative comparisons are made between data taken with low energy sources and those taken with high energy sources.

\section{ACKNOWLEDGMENTS}

The authors wish to thank Dr. R. T. Williams and Dr. J. C. Rife who relinquished some of their beam time on SURF and spent long hours assisting in those measurements. We also wish to thank Dr. D. J. Nagel, K. Hudson, and D. I. Ma for their help during the measurements and in the preparation of the manuscript

\section{REFERENCES}

1. R.C. Hughes, "Charge-Carrier Transport Phenomena in Amorphous $\mathrm{SiO}_{2}$ : Direct Mobility and Lifetime," Phys. Rev. Lett. 30, 1333 (1973).

2. O.L. Curtis,Jr., J.R. Srour, and K.Y. Chiu, "Hole and Electron Transport in $\mathrm{SiO}_{2}$ Films," Jour. Appl. Phys. 45, 4506 (1974).

3. H.E. Boesch,Jr. and J.M. McGarrity, "Charge Yield and Dose Effects in MOS Capacitors at 80K," IEEE Trans. Nucl. Sci. NS-23, 1520 (1976)

4. C.M.Dozier, D.B. Brown and J.W. Sandelin, "Dose and Dose Rate Dependence of 8080A Microprocessor Failures," IEEE Trans. Nucl. Sci. NS-27, 1299 (1980).

5. C.M. Dozier and D.B. Brown, "Photon Energy Dependence of Radiation Effects in MOS Structures," IEEE Tran. Nucl. Sci. NS27, 1694 (1980)

6. K. Unnikrishnan and M.A. Prasad, "Energy Deposition by Electrons in Argon," Rad. Res. 80, 225 (1975).

7. S. Othmer and J.R. Srour, "Electron Transport in $\mathrm{SiO}_{2}$ Films at Low Temperatures," in The Physics of MOS Insulators, Ed. by G. Lucovsky, S.T. Panelides, and F.L. Galeener, (Pergamon Press, New York, 1980) p. 49.

8. G.A. Ausman and F.B. McLean, "Electron-Hole Pair Creation Energy in $\mathrm{SiO}_{2}$," Appl. Phys. Lett. 26, 173 (1975).

9. M. Lax, "Casacade Capture of Electrons in Solids," Phys. Rev. 119, $1502(1960)$

10. A. Mozumder and J.L. Magee, "Model of Tracks of Ionizing Radiations for Reaction Mechanisms," Rad. Res. 28, 215 (1966).

11. J.L. Magee and A. Chatterjee, "Theory of the Chemical Effects of High Energy Elections," J. Phys. Chem. 82, 2219 (1978).

12. D.B. Brown and C.M. Dozier, "Electron-Hole Recombination in Irradiated $\mathrm{SiO}_{2}$ From a Microdosimetry Viewpoint," (This Issue).

13. J.S. Nagpal, "Ultraviolet Dosimetry Using Thermoluminescent Phosphors - Rate Dependence," Rad. Eff. Lett. 43, 173 (1979).

14. D.B. Brown, unpublished work

15. D.B. Brown, "Photoelectron Effects on the Dose Deposition in MOS Devices Caused by Low Energy X-Ray Sources," IEEE Trans. Nucl. Sci. NS-27, 1465 (1980)

16. J.V. Gilfrich, D.B. Brown and P.G. Burkhalter, "Integral Reflection Coefficient of X-Ray Spectrometer Crystals," Appl. Spect. 29, 322 (1975). 